\title{
COMUNICACIÓN
}

\section{CONTAMINACIÓN DE PARQUES PÚBLICOS DEL CONO NORTE CON HUEVOS DE TOXOCARA SPP.}

\author{
Virgilio La Rosa V. ${ }^{1}$, Amanda Chávez V. ${ }^{2}$ y Eva Casas A. ${ }^{2}$
}

\begin{abstract}
Toxocara spp. egg contamination in the public parks of Northern Lima on recovery of body condition in adult dogs.
\end{abstract}

\begin{abstract}
Toxocariasis is an important public health zoonosis, with ingestion of infective Toxocara spp. eggs producing both ocular and visceral larval migrant syndromes in human beings. In this study, levels of Toxocara spp. egg contamination were determined at 108 public parks in northern Lima. Soil and grass samples were collected using the double W method, during the months of July and August, 1999, when the average temperature was $18.5^{\circ} \mathrm{C}$ with $90 \%$ relative humidity. The eggs were recovered by flotation in saturated $\mathrm{CINa}$, and incubated for 30 days in a $2.5 \% \mathrm{~K}$ bicromate solution. Of the 108 parks sampled, 37 were found to be contaminated with Toxocara spp. eggs, accounting a prevalence of $34.3 \% \pm 9.0$. The highest prevalence, $64.5 \pm 16.8$, was found in well maintained parks, followed by poorly and badly maintained parks with 27.6 and $18.8 \pm 11.1$. The incidence in upper, upper middle, middle, lower middle and lower class neighborhood parks was $50.0 ; 66.7 ; 37.9 ; 25.8 \pm 15.4$ and 17.9 , respectively. Average contamination was 2.4 eggs per $100 \mathrm{~g}$ of sample. Viability of the Toxocara spp. eggs was tested by infecting 5 quail with 1500 recovered larval eggs each, with 5 quail studied as controls. The quail were culled at 24 hours, $5,10,15$ and 20 days postinoculation. Using the Baermann method, larval migrants were recovered from the liver and, less frequently, in the lungs, heart, muscle and gizzard. No larval migrants were found in the control quail. It is clear that Toxocara spp. contamination in the public parks of northern Lima represents a significant threat to public health, and it is imperative that authorities must try to control this problem at the earliest possible date.

Key words: Toxocara spp., larval migrants, zoonosis, toxocariasis.

Palabras clave: Toxocara spp., larva migrante, zoonosis, toxocariasis.
\end{abstract}

El hombre al estar en constante contacto con sus mascotas está propenso a adquirir una serie de enfermedades zoonóticas (Soulsby, 1987), debido a las malas condiciones sanitarias en que son criadas gran parte de las mascotas y los deficientes hábitos higiénicos de algunos propietarios, en especial de los niños (Botero y Restrepo, 1998). En estas mascotas se pueden encontrar una diversidad de parásitos, entre los cuales tenemos Toxocara canis, Toxascaris leonina y Toxocara cati, los que pueden causar problemas de toxocariosis y toxocariasis en el humano (Acha y Szyfres, 1988).

Generalmente esta infección se da por la ingestión de los huevos larvados con el segundo estadío de Toxocara spp. (Atías, 1994), que se encuentran diseminados en la tierra y césped de los parques públicos (Georgi y Georgi, 1994). La toxocariasis es transmitida al hombre principalmente por los

\footnotetext{
1 Práctica privada

${ }^{2}$ Laboratorio de Parasitología IVITA-FMV-UNMSM. E-mail: d170010@unmsm.edu.pe
} 
perros y con menor frecuencia por los gatos, dando lugar al Síndrome de la larva migrante visceral (LMV) (Humbert et al., 1995).

La alta prevalencia de Toxocara spp en perros y gatos, el gran número de huevos que éstos eliminan y su gran resistencia al medio ambiente, principalmente en suelos húmedos, favorecen su supervivencia y contribuyen a la contaminación del suelo el cual es la principal fuente de infección para el hombre (Acha y Szyfres, 1988).

Un alto porcentaje de casos de LMV se presentan en niños con antecedentes de pica, geofagia, como también con historia de deficiente saneamiento ambiental en las viviendas, y mala higiene personal (Botero y Restrepo, 1998).

La toxocariasis actualmente es un problema más frecuente de lo que se consideraba; debido al mayor número de perros en la ciudad y a la estrecha relación perro/persona. Esta relación es aproximadamente de 1:6 en Lima-Perú (Effio, 1998). Otros factores de importancia son la contaminación de parques públicos con huevos de Toxocara canis, el elevado número de perros parasitados que no concurren a veterinarias, la alta población de perros vagabundos infectados con Toxocara canis, y las costumbres de las personas de salir a recrearse a los parques (Saredi et al., 1995).

Se han venido realizando estudios sobre la contaminación de parques públicos con huevos de Toxocara spp. en distintas zonas de Lima Metropolitana. Guerrero (1975) reportó $24 \%$ de parques contaminados a nivel de Lima Metropolitana, y en recientes estudios, Cajas (1999) determinó un 29.6\% de positividad en los parques públicos del Cono Sur, Velarde (1999) encontró un 37\% en los parques públicos de la Provincia Constitucional del Callao, y por último Serrano (2000) determinó que el $41.1 \%$ de parques públicos del Cono Este se encontraban infestados con huevos de Toxocara spp.
El presente estudio evaluó la contaminación de los parques públicos del Cono Norte de Lima Metropolitana (Ancón, Carabayllo, Comas, Independencia, Los Olivos, Puente Piedra, Rímac, San Martín de Porres y Santa Rosa) con huevos de Toxocara spp., durante los meses de julio y agosto de 1999 , donde se tuvo una humedad relativa de $90 \%$ y una temperatura promedio de $18.5^{\circ} \mathrm{C}$ (SENAMHI, 1999). Se colectaron muestras de tierra y césped (1 a $4 \mathrm{~kg}$ ) mediante el método de la doble $\mathrm{W}$, siendo procesadas por el método de flotación con solución saturada de cloruro de sodio, considerándose positiva la muestra que presentaba uno ó más huevos de Toxocara spp. Los parques fueron clasificados según el estado de conservación y el estrato socioecónomico del barrio. La clasificación según el estado de conservación fue: bien conservados (césped en aproximadamente el $100 \%$ de su área), medianamente conservados (césped en aproximadamente el 50\% de su área) y mal conservados (césped en menos del $20 \%$ de su área). La clasificación por estrato socio económico se hizo en base a directivas del Instituto Nacional de Estadística e Informática (INEI, 1998), considerándose los siguientes estratos: Alto, medio alto, medio, medio bajo y bajo.

Para determinar la viabilidad de los huevos obtenidos de parques pósitivos, estos se incubaron en una solución de bicromato de potasio al $2.5 \%$ por 30 días con el fin de obtener huevos larvados. 1500 de estos huevos larvados fueron inoculados vía oral en 5 codomices, mientras 5 codornices se mantuvieron como control. Las codomices fueron sacrificadas a las 24 horas; 5,10 , 15 y 20 días post inoculación respectivamente para determinar lesiones macroscópicas y recuperar larvas migrantes mediante la técnica de Baermann.

Se determinó el tamaño muestral (108 parques) con la fórmula de proporciones en poblaciones finitas (Daniel, 1996), y posteriormente se estratificó el número de parques por distrito. Los resultados se expresa- 
Cuadro 1. Distribución de parques contaminados con huevos de Toxocara spp. según distrito de Lima Metropolitana 1999.

\begin{tabular}{lccc}
\hline \multicolumn{1}{c}{ Distrito } & $\begin{array}{c}\mathbf{N}^{\circ} \text { de parques } \\
\text { muestreados }\end{array}$ & $\begin{array}{c}\mathbf{N}^{\circ} \text { de parques } \\
\text { positivos }\end{array}$ & $\begin{array}{c}\text { Frecuencia } \\
(\%) \pm I . C\end{array}$ \\
\hline Ancón & 04 & 01 & 25 \\
Carabayllo & 06 & 01 & 16.7 \\
Comas & 24 & 09 & 37.5 \\
Independencia & 11 & 03 & 27.3 \\
San Martín de Porres & 24 & 08 & 33.3 \\
Los Olivos & 25 & 11 & 44 \\
Puente Piedra & 04 & 01 & 25 \\
Rímac & 09 & 03 & 33.3 \\
Santa Rosa & 01 & 00 & 00 \\
\hline Total & 108 & 37 & $34.3 \pm 9.0$ \\
\hline
\end{tabular}

ron en forma porcentual, calculándose sus respectivos intervalos de confianza, a través de la aproximación normal a la binomial.

El 34.3\% de los parques públicos del Cono Norte de Lima Metropolitana se encuentran contaminados con huevos de Toxocara spp. El distrito de Los Olivos presentó el porcentaje más alto de contaminación con un $44 \%$, mientras que el distrito de Santa Rosa no presentó contaminación, sin embargo en este distrito sólo se evaluó un parque (Cuadro 1).

Según el estado de conservación de los parques, el mayor porcentaje de parques positivos se encontró en los bien conservados $(64.5 \%)$ y los de menor contaminación fueron los mal conservados con un $\mathbf{1 8 . 8 \%}$ de positividad (Cuadro 2). Los parques bien conservados presentan áreas con mayor vegetación que proporcionarían condiciones

Cuadro 2. Distribución de parques contaminados según estado de conservación. Lima Metropolitana 1999.

\begin{tabular}{lccc}
\hline Estado de parques & $\begin{array}{c}\mathrm{N}^{\circ} \text { de parques } \\
\text { muestreados }\end{array}$ & $\begin{array}{c}\mathrm{N}^{\circ} \text { de parques } \\
\text { positivos }\end{array}$ & $\begin{array}{c}\text { Frecuencia } \\
(\%) \pm \mathrm{I} . \mathrm{C} .\end{array}$ \\
\hline Bien conservados & 31 & 20 & $64.5 \pm 16.8$ \\
Medianamente & 29 & 08 & 27.6 \\
conservados & 48 & 09 & $18.8 \pm 11.1$ \\
Mal conservados & 108 & 37 & $34.3 \pm 9.0$ \\
\hline Total & &
\end{tabular}

óptimas de humedad, temperatura y sombra que favorecen la supervivencia de los huevos de Toxocara spp., mientras que en los parques mal conservados, estos huevos estarían expuestos a la desecación y a la acción directa de los rayos solares que los destruirían en corto tiempo.
Respecto al estrato socio económico, el mayor porcentaje de parques contaminados con huevos de Toxocara spp. se encuentran en los estratos medio alto y alto respectivamente, mientras que los menos contaminados se escuentran en el estrato bajo (Cuadro 3). Estos resultados se pueden atri- 
Cuadro 3. Distribución de parques contaminados con huevos de Toxocara spp. según el estrato socioeconómico de Lima Metropolitana (INEI, 1998 ).

\begin{tabular}{|c|c|c|c|c|}
\hline Estrato & Distrito & $\begin{array}{c}\mathrm{N}^{\circ} \text { de parques } \\
\text { muestreados }\end{array}$ & $\begin{array}{c}\mathrm{N}^{\circ} \text { de parques } \\
\text { positivos }\end{array}$ & $\begin{array}{l}\text { Frecuencia } \\
(\%) \pm \text { I.C. }\end{array}$ \\
\hline Alto & $\begin{array}{l}\text { Ancón } \\
\text { Santa Rosa }\end{array}$ & 02 & 01 & 50.0 \\
\hline Medio alto & $\begin{array}{l}\text { Comas } \\
\text { Los Olivos } \\
\text { Rímac }\end{array}$ & 18 & 12 & 66.7 \\
\hline Medio & $\begin{array}{l}\text { Comas } \\
\text { Independencia } \\
\text { Los Olivos } \\
\text { Puente Piedra } \\
\text { Rímac } \\
\text { San Martín de Porres }\end{array}$ & 29 & 11 & 37.9 \\
\hline Medio bajo & $\begin{array}{l}\text { Ancón } \\
\text { Carabayllo } \\
\text { Comas } \\
\text { Independencia } \\
\text { Los Olivos } \\
\text { Puente Piedra } \\
\text { Rímac } \\
\text { San Martín de Porres }\end{array}$ & 31 & 08 & $25.8 \pm 15.4$ \\
\hline Bajo & $\begin{array}{l}\text { Ancón } \\
\text { Carabayllo } \\
\text { Comas } \\
\text { Independencia } \\
\text { Los Olivos } \\
\text { Puente Piedra } \\
\text { San Martín de Porres }\end{array}$ & 28 & 05 & 17.9 \\
\hline Total & & 108 & 37 & $34.3 \pm 9.0$ \\
\hline
\end{tabular}

buir a que en los estratos altos encontramos la mayor cantidad de parques en buen estado de conservación, los cuales favorecerían la supervivencia de los huevos de Toxocara spp. Así mismo atraen a los propietarios a sacar a sus mascotas a pasear dando lugar a que estás realizen sus deposiciones en los parques.

Adicionalmente, se evaluó la cantidad de huevos de Toxocara spp. en $100 \mathrm{~g}$ de muestra de parques positivos encontrándo- se un promedio de 2.4 huevos. Los distritos con mayor densidad de perros por parques fueron los que presentaron mayor promedio de huevos en $100 \mathrm{~g}$ de muestras como el Rímac y Comas (Cuadro 4).

Se aislaron larvas migrantes en el hígado, pulmones, corazón, músculos y proventrículo en las codornices inoculadas, lo que comprueba la viabilidad de los huevos. Así mismo se observaron ligeras lesiones macroscópicas en los órganos afectados. 
Cuadro 4. Número de huevos de Toxocara spp. en muestras de $100 \mathrm{~g}$ de parques positivos, según estado de conservación de parques del Cono Norte de Lima Metropolitana, 1999.

\begin{tabular}{lccccc}
\hline Distrito & $\begin{array}{c}\text { Bien } \\
\text { conservado }\end{array}$ & $\begin{array}{c}\text { Medianamente } \\
\text { conservado }\end{array}$ & $\begin{array}{c}\text { Mal } \\
\text { conservado }\end{array}$ & $\begin{array}{c}\text { Total por } \\
\text { distrito }\end{array}$ & Promedio \\
\hline Ancón & $\mathbf{0}$ & - & - & $\mathbf{0}$ & $\mathbf{0}$ \\
Carabayllo & $\mathbf{0}$ & - & - & $\mathbf{0}$ & $\mathbf{0}$ \\
Comas & 10 & 9 & 7 & 26 & 2.9 \\
Independencia & 1 & - & 2 & 3 & 1.0 \\
Los Olivos & 17 & 11 & $\mathbf{0}$ & 28 & 2.5 \\
Puente Piedra & 2 & - & - & 2 & 2.0 \\
Rímac & 14 & - & - & 14 & 4.7 \\
San Martín de Porres & 14 & 2 & $\mathbf{0}$ & 16 & 2.0 \\
\hline Total & 58 & 22 & $\mathbf{9}$ & $\mathbf{8 9}$ & 2.4 \\
\hline
\end{tabular}

Se concluye que existe una alta prevalencia de parques públicos contaminados con huevos de Toxocara spp. en los distritos del Cono Norte de Lima, constituyendo una importante fuente de infección para el hombre. Se debe desarrollar campañas de educación sanitaria, desparasitación de perros y gatos, controlar la población de perros y gatos, y reglamentar la circulación de perros en los parques públicos, mediante leyes y ordenanzas municipales. Por otro lado se debe informar a las autoridades sanitarias y municipales los resultados del presente estudio y del potencial peligro para la salud pública.

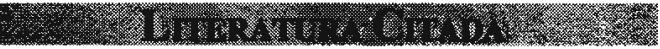

1. Acha, N.P.y B. Szyfres. 1989. Zoonosis y enfermedades transmisibles comunes al hombre y a los animales. 2da ed., Editorial OPS/OMS, EUA., p. 989.

2. Atías, A. 1994. Parasitología Clínica. 3ra ed., p:314-318, Editorial Mediterraneo, Chile.

3. Botero, D. y M. Restrepo. 1998. Parasitosis Humana.Corporación para investigaciones Biológicas. 3ra. ed., p. 335-340. Medellín, Colombia.

4. Cajas, U. 1999. Estudio de la contaminación de parques públicos con huevos de Toxocara spp. en los distritos del
Cono Sur. Tesis Médico Veterinario, FMV-UNMSM., pp. 77.

5. Daniel, W. 1996. Biostadística. Base para el análisis de las ciencias de la salud. 3ra. ed., p. 206. Editorial LIMUSA S.A., México.

6. Effio, J.F. 1998. Estudio preliminar del mercado veterinario peruano. INDECOPI. Lima-Perú. Fuente de la cantidad de perros del Cono Norte de Lima Metropolitana, p. 258.

7. Georgi, J.R. y M.E. Georgi. 1994. Parasitología en clínica canina. 4a. ed., p.171-178, Editorial Interamericana, México.

8. Guerrero, M.O. 1975. Estudio de la contaminación de parques públicos de Lima Metropolitana con huevos de Toxocara spp. Tesis Médico Veterinario, FMVUNMSM. Lima-Perú. 12 pp.

9. Humbert, P.; A. Buchet y T. Barde. 1995. Toxocariasis: A cosmopolitan parasitic zoonosis. Allerg Inmunol (Paris) 27(8): 284-291.

10. Instituto Nacional de Estadística e Informática (INEI). 1998. Planos estratificados de Lima Metropolitana a nivel de manzanas según estratos socioeconómicos de hogares. Bol. De estadísticas geográficas. 9700.002 (1): 10 45. 
11. Saredi, N; E. Mavromatupulos; N. Castagnino y C. Depalma. 1995. Toxocariasis en Pediatría, Hallazgos Clínicos y de Laboratorio. Rev. Soc. Chil. Parasitol, 19: 359.

12. SENAMHI, 1999. Pronóstico Meteorológico Mensual para Lima, julio-agosto, p. 1-13.

13. Serrano, M.E. 2000. Estudio de la contaminación de los parques públicos con huevos de Toxocara spp. en los distritos del Cono Este. Tesis Médico Veterinario. FMV-UNMSM. 73 pp.

14. Soulsby, E.J. 1987. Parasitología y enfermedades parasitarias de los animales domésticos. 7a. ed., p. 150-155. Editorial Interamericana S.A. México.

15. Velarde, J.A. 1999. Contaminación de los parques públicos de la Provincia Constitucional del Callao con huevos de Toxocara spp. Tesis Médico Veterinario. FMV-UNMSM., pp. 62. 